\title{
A hybrid framework of multiple active appearance models and global registration for 3D prostate segmentation in MRI
}

\author{
Soumya Ghose ${ }^{a b}$, Arnau Oliver ${ }^{a}$, Robert Martía, Xavier Lladó ${ }^{a}$, Jordi Freixenet ${ }^{a}$, Jhimli \\ Mitra $^{a b}$, Joan C. Vilanova ${ }^{c}$ and Fabrice Meriaudeau ${ }^{b}$ \\ ${ }^{a}$ Universitat de Girona, Computer Vision and Robotics Group, Girona, Spain. \\ ${ }^{b}$ Université de Bourgogne, Le2i-UMR CNRS 5158, Le Creusot, Bourgogne, France. \\ ${ }^{c}$ Clnica Girona, Carrer Joan Maragall, Girona, Catalonia, Spain.
}

\begin{abstract}
Real-time fusion of Magnetic Resonance (MR) and Trans Rectal Ultra Sound (TRUS) images aid in the localization of malignant tissues in TRUS guided prostate biopsy. Registration performed on segmented contours of the prostate reduces computational complexity and improves the multimodal registration accuracy. However, accurate and computationally efficient 3D segmentation of the prostate in MR images could be a challenging task due to inter-patient shape and intensity variability of the prostate gland. In this work, we propose to use multiple statistical shape and appearance models to segment the prostate in $2 \mathrm{D}$ and a global registration framework to impose shape restriction in 3D. Multiple mean parametric models of the shape and appearance corresponding to the apex, central and base regions of the prostate gland are derived from principal component analysis (PCA) of prior shape and intensity information of the prostate from the training data. The estimated parameters are then modified with the prior knowledge of the optimization space to achieve segmentation in $2 \mathrm{D}$. The 2D segmented slices are then rigidly registered with the average 3D model produced by affine registration of the ground truth of the training datasets to minimize pose variations and impose 3D shape restriction. The proposed method achieves a mean Dice similarity coefficient (DSC) value of $0.88 \pm 0.11$, and mean Hausdorff distance (HD) of $3.38 \pm 2.81 \mathrm{~mm}$ when validated with 15 prostate volumes of a public dataset in leave-one-out validation framework. The results achieved are better compared to some of the works in the literature.
\end{abstract}

Keywords: Prostate Cancer, MRI, 3D Prostate Segmentation, Active Appearance Model

\section{INTRODUCTION}

Prostate cancer is one of the most commonly diagnosed cancer in North America accounting for over 33,000 estimated deaths in 2011. ${ }^{1}$ In TRUS guided needle biopsy, localization of malignant tissues is difficult due to the low soft tissue contrast. The localization of malignant tissues could be improved by real-time registration of the 2D TRUS video sequence with 3D pre-acquired MRI during TRUS guided biopsy. ${ }^{2}$ Segmentation of the prostate in TRUS and MRI aids in designing computationally efficient and accurate registration procedures ${ }^{3}$ necessary for such a procedure. However, computer aided prostate segmentation in MR images is a challenging task due to inter-patient shape and size variability of the prostate. Also, the intensity inside the prostate gland

Further author information: (Send correspondence to Soumya Ghose or Dr. Arnau Oliver or Prof. Fabrice Meriaudeau.)

Soumya Ghose: E-mail: soumyaghose@gmail.com,

Arnau Oliver: E-mail: aoliver@eia.udg.edu, Telephone: +34 972418878

Xavier Lladó: E-mail: llado@eia.udg.edu,

Robert Martí: E-mail: marly@eia.udg.edu,

Jordi Freixenet: E-mail: jordif@eia.udg.edu,

Joan C. Vilanova: E-mail: kvilanova@comg.cat,

Jhimli Mitra: E-mail: jhimlimitra@yahoo.com,

Fabrice Meriaudeau: E-mail: fabrice.meriaudeau@u-bourgogne.fr, Telephone: +33(0)385731077

Medical Imaging 2012: Image Processing, edited by David R. Haynor, Sébastien Ourselin,

Proc. of SPIE Vol. 8314, 83140S · C 2011 SPIE · CCC code: 0277-786X/11/\$18 · doi: 10.1117/12.911253

Proc. of SPIE Vol. 8314 83140S-1 
is heterogeneous and may vary depending on the MRI acquisition parameters and use of surface or endo-rectal coil. Considerable shape and intensity variability inhibits the design of a global model for the prostate.

To address the challenges of prostate segmentation in MR images Tsai et al. ${ }^{4}$ proposed to use prior prostate shape and intensity information in a levelset framework for 3D segmentation of the prostate. Shape space was considered to be Gaussian and region based intensity information was minimized with respect to the pose parameters to achieve segmentation. Similarly, Gao et al. ${ }^{5}$ used particle filters to register clouds of points created from prostate volumes to a common reference to minimize the difference in pose to determine shape prior information. Shape priors and local image statistics were incorporated in an energy function that was minimized to achieve prostate segmentation in a level set framework. In recent years atlas based segmentation of the prostate has become popular. ${ }^{6,7}$ Klein et al. ${ }^{6}$ used B-spline based non rigid registration to build prostate atlas from the training images and used the registration framework to segment prostate. Martin et al. ${ }^{7}$ used a hybrid framework of atlas and deformable model built from prior prostate shape information to segment the prostate in 3D. More recently Martin et al. ${ }^{8}$ have used probabilistic atlas and a deformable model to segment the prostate.

It has been demonstrated by Tsai et al., ${ }^{9}$ Martin et al. ${ }^{7}$ and Gao et al. ${ }^{5}$ that incorporating shape and intensity prior information in prostate segmentation methods improve the segmentation accuracy. Cootes et al. ${ }^{10}$ provided an efficient framework for combining shape and intensity prior in their Active Appearance Model (AAM). To address the challenges involved with prostate segmentation in 3D MR images, we propose a novel hybrid framework based on using 2D multiple statistical shape and appearance models and a 3D registration framework. The objective of multiple 2D statistical shape and appearance model or AAM is to improve local 2D slice segmentation accuracies for the apex, central and the base regions of the prostate. It is to be noted that, prostate segmentation is a challenging task in the base and the apex slices due to low contrast of the prostate in images. However, we build separate models for each of these zones that incorporate region-based and shapebased information for each of these regions to learn the local variability, thereby, producing a more accurate segmentation. 3D shape restriction is imposed by rigidly registering the obtained volume to a 3D average model of the prostate. The performance of our method is validated with 15 prostate public datasets ${ }^{11}$ in a leave-one-out validation framework. Experimental results show that our method achieves accurate prostate segmentation and performs better compared to some of the works in the literature which use the same datasets. ${ }^{5,12,13}$ The key contributions of this work are:

- Use of separate mean AAM for the base, central and the apex regions and use of multiple AAM for each region to improve on segmentation accuracies.

- The hybrid schema of applying AAM for 2D segmentation and a global shape restriction with 3D rigid registration.

The rest of the paper is organized as follows. Multiple AAM of the apex, central and the base region and the $3 \mathrm{D}$ registration framework is formulated in Section 2 followed by quantitative and qualitative evaluation of our method in Section 3. We finally draw conclusion in Section 4.

\section{METHOD}

The proposed method illustrated in Fig. 1, is developed on two major components, (1) adaptation of traditional $\mathrm{AAM}^{10}$ to produce multiple mean models of the apex, central and the base regions of the prostate and (2) use of a global registration schema to impose 3D shape restriction. Traditional AAM is presented first, followed by a comprehensive discussion about multiple AAM to segment the prostate. The global registration framework is presented thereafter. 


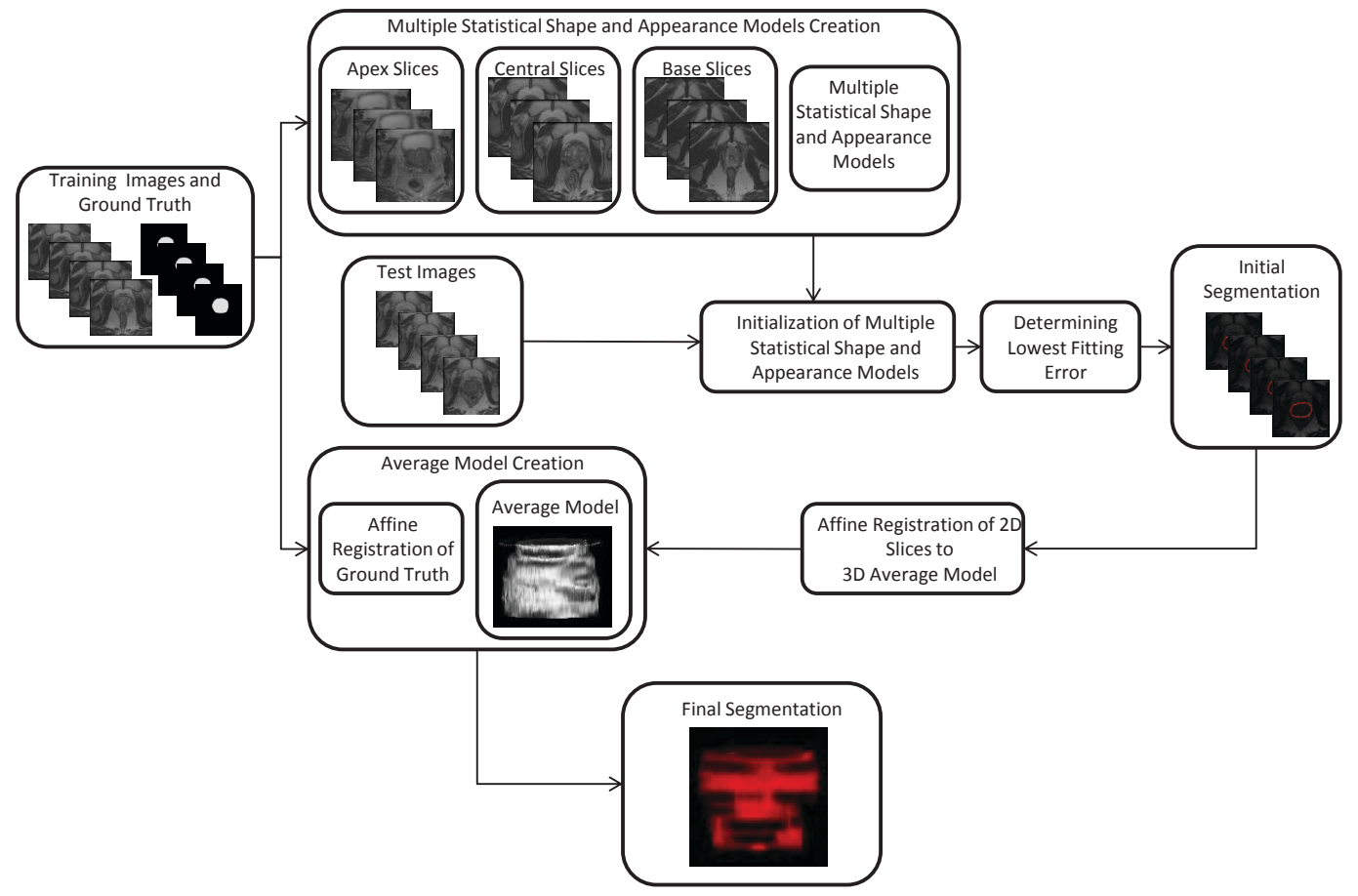

Figure 1. Schematic representation of our approach. The final segmentation is given in red contour and ground truth in green.

\subsection{Active Appearance Model}

In traditional AAM, PCA of the point distribution models ${ }^{10}$ of the manually segmented contours aligned to a common reference frame by generalized Procrustes analysis is used to identify the principal modes of shape variations. PCA of intensity distributions warped into correspondence using a piece-wise affine warp and sampled from shape free reference, is used to identify the principal components of intensity variations. The shape and the intensity model may be formalized in the following manner. Let $E\{s\}$ and $E\{t\}$ represent the shape and intensity models of AAM, where $s$ and $t$ are the shape and intensities of the corresponding training images, $\bar{s}$ and $\bar{t}$ are the mean shape and mean intensity, $\phi_{s}$ and $\phi_{t}$ are the truncated eigenvector matrices of shape and intensity respectively (obtained from $98 \%$ of the total variations to identify the primary components of the variations), and $\theta_{s}$ and $\theta_{t}$ are the corresponding deformation parameters.

$$
\begin{array}{r}
E\{s\}=\bar{s}+\phi_{s} \theta_{s} \\
E\{t\}=\bar{t}+\phi_{t} \theta_{t}
\end{array}
$$

The shape and intensity model are combined in a linear framework to give the combined model $b$ as,

$$
b=\left[\begin{array}{c}
W \theta_{s} \\
\theta_{t}
\end{array}\right]=\left[\begin{array}{c}
W \phi_{s}^{T}(E\{s\}-\bar{s}) \\
\phi_{t}^{T}(E\{t\}-\bar{t})
\end{array}\right]
$$

where $W$ denotes a weight factor (determined as in $\mathrm{AAM}^{10}$ ) coupling the intensity and the shape space. A third PCA of the combined model removes redundancy in the combined model giving $\widehat{b}$ as,

$$
\widehat{b}=V c
$$

where $V$ is the matrix of eigenvectors and $c$ the appearance parameters. Given a test image, the sum of squared difference of the intensities between the test image and mean model is minimized with respect to the 
pose (translation, rotation and scaling) parameters. Prior knowledge of the optimization space is acquired by perturbing the combined model and the pose parameter with some known values and recording the corresponding changes in the intensities. A linear relationship between the known perturbation of the combined model $(\delta c)$ and know perturbation of the pose parameters $(\delta p)$ and the residual intensity values $(\delta t)$ (obtained from sum of squared difference between the intensities of the perturbed mean model and the target image) are acquired in a multivariate regression framework as,

$$
\delta c=R_{c} \delta t, \quad \delta p=R_{p} \delta t
$$

where $R_{c}$ and $R_{p}$ refer to the correlation coefficients. Given a new instance, equation (4) is used as update parameters where residual intensity value $(\delta t)$ is used to generate new pose parameters, new model parameters and hence new intensity values. The process continues in an iterative manner until the differences with the target image remains unchanged.

AAM assumes the shape space, the intensity space and hence the combined model space to be Gaussian. However, inter-patient prostate shape and intensity may vary significantly. Moreover prostate shape and intensity values varies across the base, the central and the apex regions of the prostate and under such circumstances approximating with a single Gaussian mean AAM introduces segmentation inaccuracies. To address this problem we propose to use multiple 2D AAM. The objective of multiple 2D AAM is to improve local 2D slice segmentation accuracies for the apex, central and the base regions of the prostate. Prostate segmentation is a challenging task in the base and the apex slices due to low contrast of the prostate in the images. Therefore, we build separate models for the apex, central and the base regions that incorporate region-based and shape-based information for each of these regions to learn the local variabilities.

The schema for building the multiple models for the apex, central and the base regions is as follows; initially the prostate slices of the training volumes are divided into three distinct sections the apex, the central region and the base. For dividing the prostate the number of slices of prostate (obtained from ground truth values) is divided by 3 , the resulting quotient is used to group the slices from the top and the bottom into apex and the base groups and remaining slices of the central region are placed in one group. The objective of such grouping is to produce different mean models for each of these regions that approximate each of these regions better and improve segmentation accuracies.

Moreover for a given region (apex, central and base) multiple mean models are produced to better approximate local variability of each of the region. The sum of squared differences of the intensities between a mean model and the target image is recorded as fitting or registration error after the final segmentation with each of the mean model of all regions (apex, central and base). The segmentation result of the mean model with least fitting error is considered as the optimized segmentation for that particular image. The framework of building multiple mean models for each of the region (apex, central, base) is as follows; the base region has 43 slices from 15 datasets. Initially slice 1 is selected as the reference to register slices 3 to 43 to build the mean model and test it on slice 2 and record the fitting error (sum of squared distance of the intensity between the mean model and the test image, slice 2). Likewise, with the fixed reference (slice 1) we build the second mean model by registering slice 2 and slices, 4-43 to test slice 3 and record the fitting error. The process is repeated for all the slices to generate 42 model fitting error with slice 1 as reference as shown in Fig. 2.

Consequently the reference dataset is changed from 2-43 to generate 43 model fitting error graphs (one for each slice). We have analyzed the model fitting error values and corresponding segmentation accuracies and have observed that less fitting error translates into higher segmentation accuracies (in terms of DSC, HD etc). An empirical error value is determined from the 43 model fitting error graph (the red line $\geq 3000$ in our case) beyond which the segmentation accuracy is reduced. The reference slice that has fitting error less than this empirical value with maximum number of slices is selected, grouped together (slice 1, 4, 14, 16, 20, 27, and 41) and removed from further grouping. The process is repeated until all the slices are grouped. These groups of datasets provide individual mean models ( 8 mean models in our case). However, increasing the number of mean models (decreasing the fitting error threshold) improves segmentation accuracy with additional computational time. Hence, the choice of optimum number of mean models depends on the segmentation accuracy and computational 


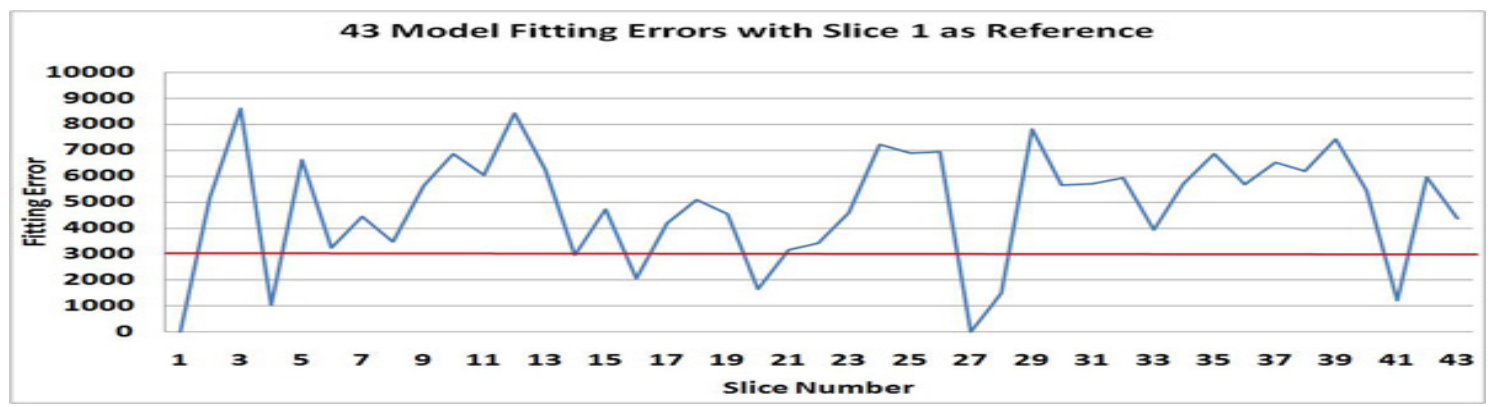

Figure 2. Mean models fitting errors with dataset 1 as reference.

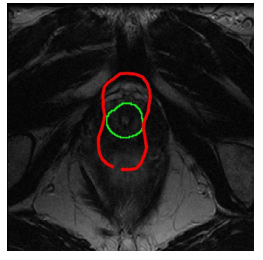

(a)

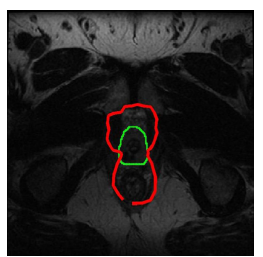

(g)

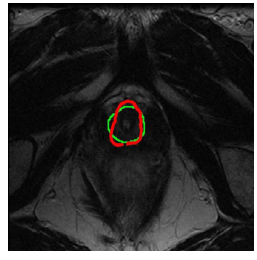

(b)

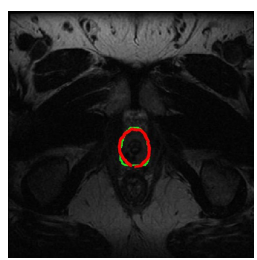

(h)

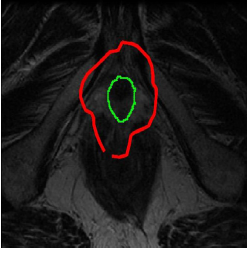

(c)

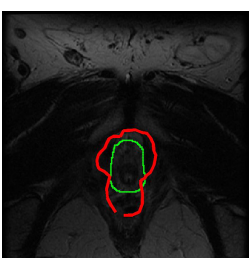

(i)

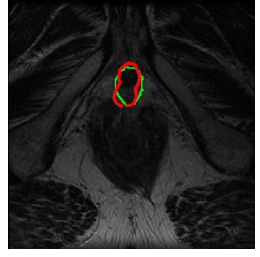

(d)

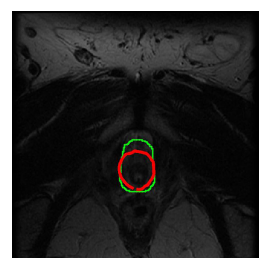

(j)

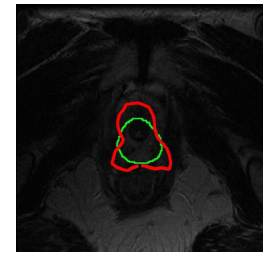

(e)

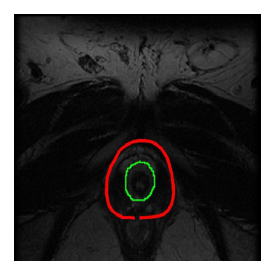

(k)

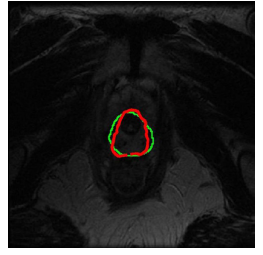

(f)

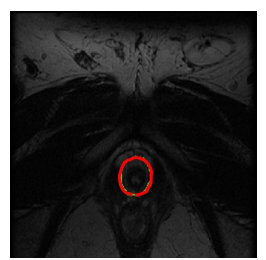

(1)

Figure 3. (a),(c), (e), (g), (i), (k), segmentation without multiple mean models, (b),(d), (f), (h), (j), (l), segmentation with multiple mean models. The green contour gives the ground truth and the red contour gives the obtained result of some of the base slices.

time requirement of the process. The effect of building multiple mean models could be observed in Fig. 3 . In Fig. 3 green contour gives the ground truth and the red contour shows the achieved segmentation. Fig. (a),(c), (e), (g), (i), and (k) show segmentation achieved in some base slices prior to the use of multiple mean models. Fig. (b), (d), (f), (h), (j), and (l) show segmentation achieved for the same slices with multiple mean models.

\subsection{Average 3D Model}

The process of construction of an average $3 \mathrm{D}$ model of the prostate begins with alignment of $N$ manually segmented training dataset to a common reference. One among $N$ training labeled datasets is manually selected by an expert and $N-1$ labeled datasets are registered to the reference dataset. Intensity based affine registration of $N-1$ datasets to the reference dataset is employed to create an average 3D model of the prostate. Effect of intensity based affine registration on the overlap of the ground truth is illustrated in Fig. 4 and the average model created in the process is illustrated in Fig. 5. Given a test patient dataset multiple AAM is employed to achieve 2D slice by slice segmentation of the dataset. A 3D model of the labels is created from 2D segmented slices of the test dataset. The average 3D model (Fig. 5) built from training images is re-sampled using cubic interpolation to produce equal number of slices as the test dataset. The 3D model of the labels of the test dataset is then translated to the center of gravity of the 3D average shape model and then rotated to minimize the pose differences and impose 3D restrictions.

\section{RESULTS}

We have validated the accuracy and robustness of our approach with $15 \mathrm{MR}$ public datasets with image resolution of $256 \times 256$ from the MICCAI prostate challenge ${ }^{11}$ in leave-one-out evaluation strategy. During validation the 


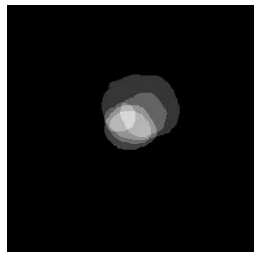

(a)

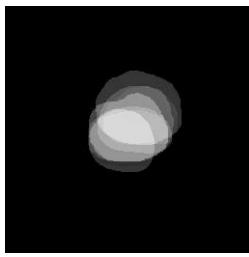

(g)

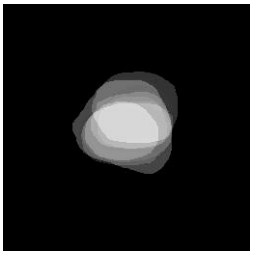

$(\mathrm{m})$

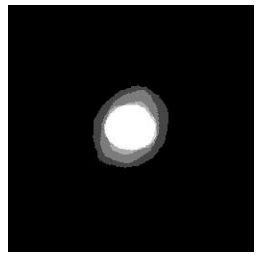

(b)

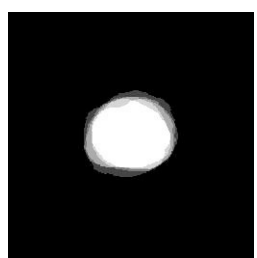

(h)



(n)

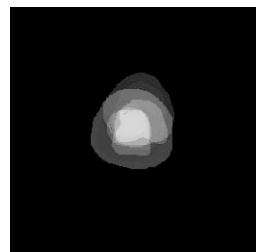

$(\mathrm{s})$

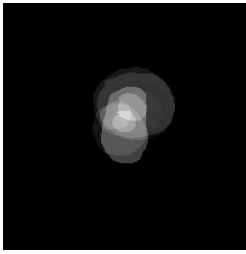

(c)

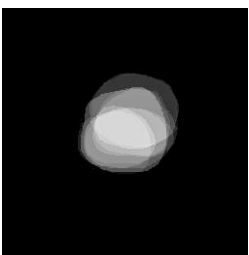

(i)

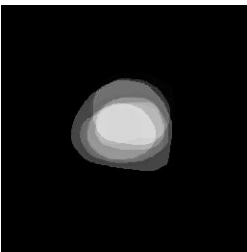

(o)

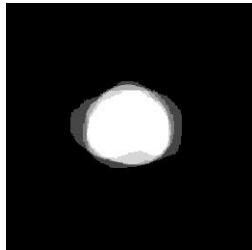

$(\mathrm{t})$

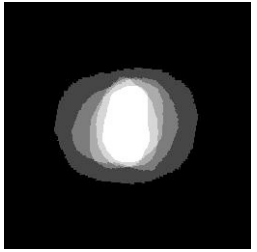

(d)

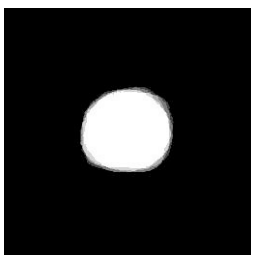

(j)

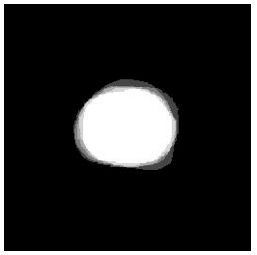

(p)

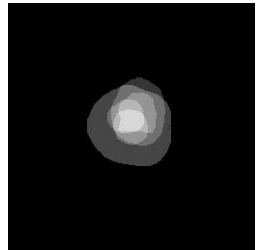

(u)

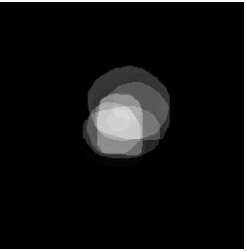

(e)

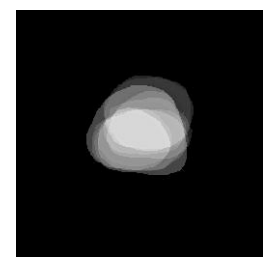

(k)

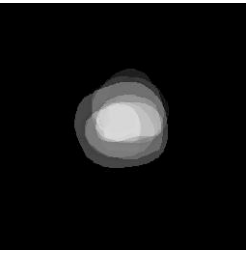

(q)

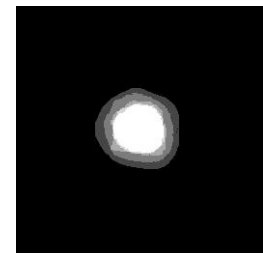

$(\mathrm{v})$

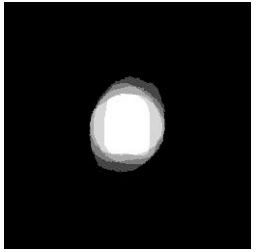

(f)

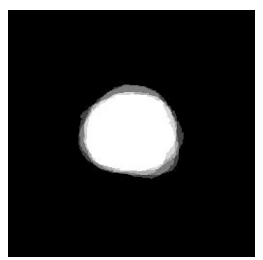

(1)

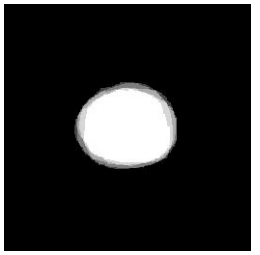

(r)

Figure 4. Illustration of effect of intensity based 3D affine registration. (a), (c), (e), (g), (i), (k), (m), (o), (q), (s), (u) Shows overlapping slices of 14 datasets before registration and (b), (d), (f), (h), (j), (k), (n), (p), (r), (t), (v) shows corresponding overlapping slices after registration to a common reference.

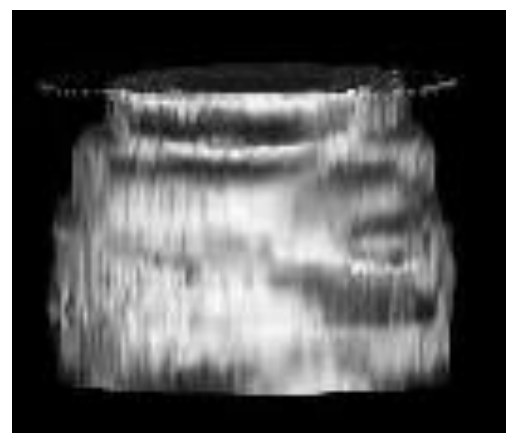

Figure 5. Average model created from the training datasets.

test dataset is removed and multiple mean models of the apex, central and the base regions are developed with the remaining 14 datasets. An average 3D model is also built with the 14 datasets. The segmentation process is initialized by clicking only on the center of a central slice of the test dataset. All the mean models of all sections (apex, central and base) are applied to segment the slice. The segmentation result of the mean model producing the least fitting error is selected. The center of gravity of the segmented mask is used to initialize segmentation 

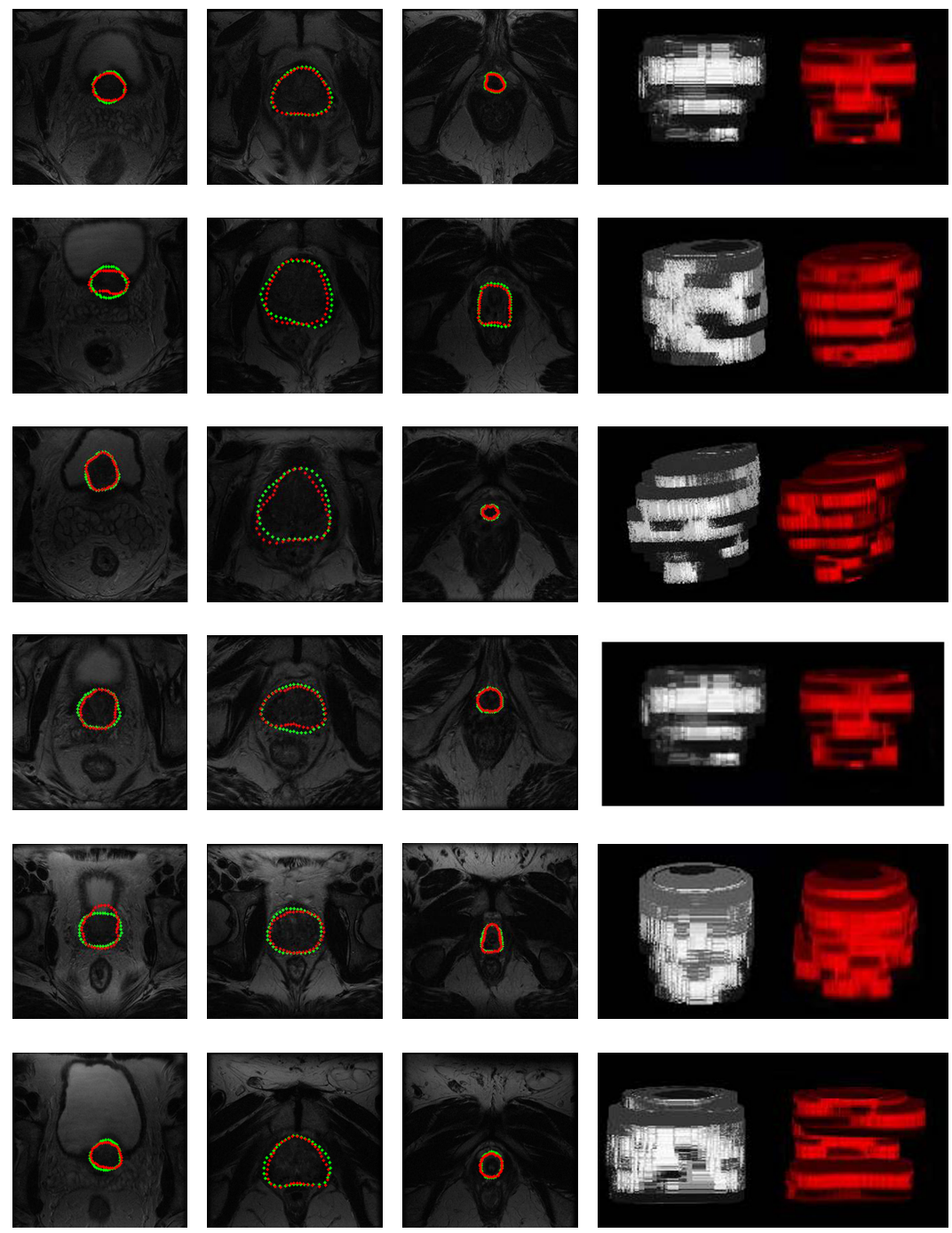

Figure 6. Subset of segmentation results of 6 datasets. One axial slice from the apex, base and the central regions are displayed. First column shows apex slices, second row shows slices from the central region and the third column shows base slices and fourth column shows volume created from the ground truth and achieved segmentation. Green contour shows the ground truth and red contour shows the achieved segmentation. The white volume is created from the ground truth and red volume is created from achieved segmentation where number of slices varies from 8 to 14 .

in slices above and below the central slice and the process is repeated until all the slices are segmented. Our AAM optimization space is created that supports translation of \pm 5 pixels, rotation of \pm 10 degrees and scale of $\pm 5 \%$. Such an optimization space is useful to compensate initialization error of the model away from the prostate center either due to slice propagation or due to human error in initialization. Qualitative results of our approach are presented in Fig. 6. We have used most of the popular prostate segmentation evaluation metrics like DSC, 95\%, HD, Mean Absolute Distance (MAD), Maximum Distance (MaxD), specificity, sensitivity, and accuracy to evaluate our method. Quantitative results are given in Table 1.

To have an overall qualitative estimate of our performance we have compared our method with results published in MICCAI prostate challenge 2009, ${ }^{1213}$ and with the work of Gao et al. ${ }^{5}$ in Table 1 . We observe that our method performs better than some of the works in literature. Observing Table 1 we can see that in general our method performs well compared to,${ }^{1213}$ and. ${ }^{5}$ The improved overlap (DSC) and contour (HD) accuracy may 
Table 1. Prostate segmentation quantitative results $($ secs $=$ seconds, mins $=$ minutes, vol. $=$ volume, $\mathrm{mm}=$ millimeter, pxs $=$ pixels)

\begin{tabular}{|l|l|l|l|l|l|l|l|}
\hline Method & DSC & HD & MAD & Specificity & Sensitivity & Accuracy & Time \\
\hline Merida et al. $^{\mathbf{1 2}}$ & 0.79 & $7.11 \mathrm{~mm}$ & - & - & - & - & $60 \mathrm{mins} / \mathrm{vol}$. \\
Dowling et al. $^{\mathbf{1 3}}$ & $0.73 \pm 0.11$ & - & - & - & - & - & $60 \mathrm{mins} / \mathrm{vol}$. \\
Gao et al. $^{\mathbf{5}}$ & $0.82 \pm 0.05$ & $10.22 \pm 4.03$ & - & - & - & - & - \\
\hline Our Method & $0.88 \pm 0.11$ & $3.38 \pm 2.81 /$ & $1.32 \pm 1.53 \mathrm{~mm}$ & $0.87 \pm 0.08$ & $0.996 \pm 0.006$ & $0.98 \pm 0.07$ & $43.22 \mathrm{secs} / \mathrm{vol}$ \\
& & $6.18 \pm 5.15$ & & & & \\
& & & & & \\
\end{tabular}

be attributed to our schema of multiple Gaussian mean models and global registration framework. Also, offline optimization framework of AAM significantly improves mean segmentation time. Our method was implemented in Matlab 7 on an Intel Quad Core Q9550 processor of 2.83 Ghz processor speed and 8 GB RAM. The mean segmentation time of the procedure is 43.21 seconds.

\section{CONCLUSION AND FUTURE WORKS}

A novel approach based on multiple Gaussian statistical models of shape and appearance has been proposed with the goal of segmenting a 3D prostate in MR images. Our approach produces accurate segmentation in presence of large scale shape and intensity variations. However, the method has to be validated with larger number of datasets. The proposed method could be improved further by improving on the initialization schema for the multiple AAM. Use of probabilistic atlas to obtain an initial segmentation for initialization and propagation of our multiple AAM would improve the results further.

\section{ACKNOWLEDGMENTS}

This research has been funded by VALTEC 08-1-0039 of Generalitat de Catalunya, Spain and Conseil Régional de Bourgogne, France and was partially supported by the Spanish Science and Innovation grant nb. TIN2011-23704.

\section{REFERENCES}

[1] "Prostate Cancer." American Cancer Society Atlanta, GA [Online]. Available: http://www.cancer.org/, accessed on [28th June, 2011] (2011).

[2] Xu, S., Kruecker, J., Turkbey, B., Glossop, N., Singh, A., Chyke, P., Pinto, P., and Wood, B., "Real-time MRI/TRUS Fusion for Guidance of Targeted Prostate Biopsies.," Computer Aided Surgery 13, 255-264 (2008).

[3] Yan, P., Xu, S., Turkbey, B., and Kruecker, J., "Optimal Search Guided by Partial Active Shape Model for Prostate Segmentation in TRUS Images," Proceedings of the SPIE Medical Imaging : Visualization, Image-Guided Procedures, and Modeling 7261, 72611G-72611G-11 (2009).

[4] Tsai, A., Yezzi, A., Wells, W., Tempany, C., Tucker, D., Fan, A., Grimson, W. E., and Willsky, A., "A Shape-Based Approach to the Segmentation of Medical Imagery Using Level Sets," IEEE Transactions on Medical Imaging 22, 137-154 (2003).

[5] Gao, Y., Sandhu, R., Fichtinger, G., and Tannenbaum, A. R., "A Coupled Global Registration and Segmentation Framework with Application to Magnetic Resonance Prostate Imagery," IEEE Transactions on Medical Imaging 10, 17-81 (2010).

[6] Klein, S., van der Heide, U. A., Lipps, I. M., Vulpen, M. V., Staring, M., and Pluim, J. P. W., "Automatic Segmentation of the Prostate in 3D MR Images by Atlas Matching Using Localized Mutual Information," Medical Physics 35, 1407-1417 (2008).

[7] Martin, S., Daanen, V., and Troccaz, J., "Atlas-based Prostate Segmentation Using an Hybrid Registration," International Journal of Computer Assisted Radiology and Surgery 3, 485-492 (2008). 
[8] Martin, S., Troccaz, J., and Daanen, V., "Automated Segmentation of the Prostate in 3D MR Images Using a Probabilistic Atlas and a Spatially Constrained Deformable Model," Medical Physics 37, 1579 - 1590 (2010).

[9] Tsai, A., Wells, W. M., Tempany, C., Grimson, E., and Willsky, A. S., "Coupled Multi-shape Model and Mutual Information for Medical Image Segmentation," in [International Conference, Information Processing in Medical Imaging], Taylor, C. and Noble, J. A., eds., 185-197, Springer, Berlin and Heidelberg and New York (2003).

[10] Cootes, T., Edwards, G., and Taylor, C., "Active Appearance Models," in [In Proceedings of European Conference on Computer Vision], H.Burkhardt and Neumann, B., eds., 484-498, Springer, Berlin and Heidelberg and New York (1998).

[11] "2009 prostate segmentation challenge MICCAI." Available: http://wiki.na-mic.org/Wiki/index.php, Accessed on [20th July, 2011] (2009).

[12] Gubern-Mérida, A. and Martí, R., "Atlas based segmentation of the prostate in mr images." www.wiki.namic.org/Wiki/images/d/d3/Gubern-Merida_Paper.pdf, Accessed on [20th July, 2011] (2009).

[13] Dowling, J., Fripp, J., Greer, P., Ourselin, S., and Salvado, O., "Automatic atlas-based segmentation of the prostate." www.wiki.na-mic.org/Wiki/images/f/f1/Dowling_2009 _MICCAIProstate_v2.pdf, Accessed on [20th July, 2011] (2009). 